\title{
Instrumentos de Avaliação da Sobrecarga em Cuidadores Informais: Uma Revisão Integrativa
}

\author{
Sabrina Martins Barroso ${ }^{1}$, Luísa Parreira Santos, Luciana Francielle e Silva \\ Universidade Federal do Triângulo Mineiro, Uberaba-MG, Brasil
}

\section{RESUMO}

Os cuidadores informais podem vivenciar sobrecarga devido às atividades que desempenham. A presente revisão integrativa da literatura analisou artigos publicados entre janeiro de 2002 e dezembro de 2016 visando investigar os instrumentos utilizados para avaliação da sobrecarga dos cuidadores informais de pessoas com patologias crônicas em contexto brasileiro. Pesquisou-se as bases de dados SciELO, PubMed, LILACS e Portal de Periódicos da Capes, permanecendo no escopo final 136 artigos. Os resultados mostraram 16 instrumentos/técnicas de avaliação da sobrecarga e aumento das pesquisas ao longo dos anos, com média de 9,71 artigos por ano e maior produção sobre o tema em 2012.

Palavras-chave: sobrecarga; cuidadores; instrumentos; escalas.

\section{ABSTRACT - Instruments to Evaluate Overload in Informal Caregivers: An Integrative Review}

Informal caregivers may experience overload due to the activities they perform. The present integrative literature review analyzed articles published between January 2002 and December 2016 aiming to investigate the instruments used to assess the overload of informal caregivers to people with chronic pathologies in the Brazilian context. We searched the databases SciELO, PubMed, LILACS and Capes Periodicals, narrowing the final scope to 136 articles. Results showed 16 overload evaluation instruments/techniques and an increase in surveys over the years, with a mean of 9.71 articles per year and greater production on the theme in 2012.

Keywords: overload; caregivers; instruments; scales.

\section{RESUMEN - Instrumentos de Evaluación de Sobrecarga en Cuidadores Informales: una Revisón Integrativa}

Los cuidadores informales pueden experimentar sobrecarga debido a las actividades que desempeñan. Esta revisión integrativa de literatura analizó artículos publicados entre enero de 2002 y diciembre de 2016, con el fin de investigar los instrumentos utilizados para la evaluación de sobrecarga de cuidadores informales de personas con patologías crónicas en el contexto brasileño. Se investigaron las bases de datos SciELO, PubMed, LILACS y Portal de Periódicos de Capes, permaneciendo en el objetivo final 136 artículos. Los resultados mostraron 16 instrumentos/técnicas de evaluación de sobrecarga y aumento de las investigaciones a lo largo de los años, con media de 9,71 artículos por año y mayor producción sobre el tema en 2012.

Palabras-clave: sobrecarga; cuidadores; instrumentos; escalas.

O interesse pela sobrecarga dos cuidadores informais de pessoas com patologias começou desde a década de 1960, mas só a partir da década de 1980 surgiram estudos sistemáticos internacionalmente, sendo sua avaliação ainda mais recente no Brasil (Almeida, Martins, \& Moderna, 2010; Galera, Zanetti, Ferreira, Giacon, \& Cardoso, 2011; Hedler, Santos, Faleiros, \& Almeida, 2016). A sobrecarga pode ser definida como o excesso de atividades desempenhadas pelos cuidadores (sobrecarga objetiva) ou o sentimento que as atividades desenvolvidas são demasiadas e geram sofrimento (Galera et al., 2011).

Internacionalmente, a maior parte dos estudos que avaliam a sobrecarga dos cuidadores informais utilizam instrumentos de medida validados (Wijngaarden \& Schene, 2010). Na revisão de literatura conduzida por Whalen e Buchholz (2009), foram identificados 74 instrumentos na Europa, Ásia e Américas e indicaram que a Zarit Burden Interview (ZBI), a Caregiver Reaction Assessment e o Caregiver Burden Inventory foram os instrumentos mais frequentemente utilizados. Especificamente para avaliar a sobrecarga de cuidadores de pessoas com transtornos mentais, Wijngaarden e Schene (2010) identificaram 11 instrumentos validados em diferentes partes do mundo, sendo eles: Family Burden Interview Schedule (FBIS), Social Behaviour Assessment Schedule, Family Burden Scale, Family Burden Questionnaire, Significant Other Scale, Family Problems Questionnaire, Involvement Evaluation Questionnaire, Burden 
Assessment Scale, Experience of Caregiving Inventory, Perceived Family Burden Scale, Interview Schedule for Families and Relatives of Severely Mentally Ill Person.

Há uma dificuldade em compreender o panorama brasileiro sobre a sobrecarga dos cuidadores dada a variação nos métodos utilizados para avaliá-la, que vão desde instrumentos validados até diários de campo e a falta de sistematização sobre os achados e os instrumentos utilizados. Assim, o presente trabalho investigou os instrumentos utilizados para avaliação da sobrecarga dos cuidadores informais em contexto brasileiro, por meio de uma revisão integrativa de literatura.

\section{Método}

Tipo de estudo. Revisão integrativa de literatura, baseada na pergunta "Que instrumentos foram utilizados no Brasil, entre 2002 e 2016, para avaliar a sobrecarga dos cuidadores informais de pessoas com patologias crônicas?".

Bases de dados e critérios de busca. As bases consultadas foram SciELO, PubMed, LILACS e Portal de Periódicos da Capes. As buscas foram conduzidas em janeiro de 2017. Os unitermos utilizados foram: sobrecarga, sobrecarga familiar, sobrecarga da família, sobrecarga de cuidadores, impacto familiar, carga familiar e seus correspondentes em inglês. Não houve restrição inicial quanto a patologia/causa que gerou a necessidade de um cuidador.

Os critérios de inclusão foram: 1. Trabalho empírico; 2. Amostra brasileira; 3. Publicação entre 01 de janeiro de 2002 e 31 de dezembro de 2016; 4. Trabalho completo disponível on-line; 5. Amostra de cuidadores informais.

Critérios analisados. Foram avaliados o tipo de estudo, instrumentos de mensuração da sobrecarga, tipo e tamanho da amostra e ano de publicação dos trabalhos. Os resultados foram apresentados de forma descritiva e comentados criticamente. A Tabela 1 apresenta os itens investigados e alguns exemplos que ilustram os resultados.

\section{Resultados}

O percurso de seleção dos artigos está apresentado na Figura 1. Permaneceram no escopo final da revisão 136 trabalhos.

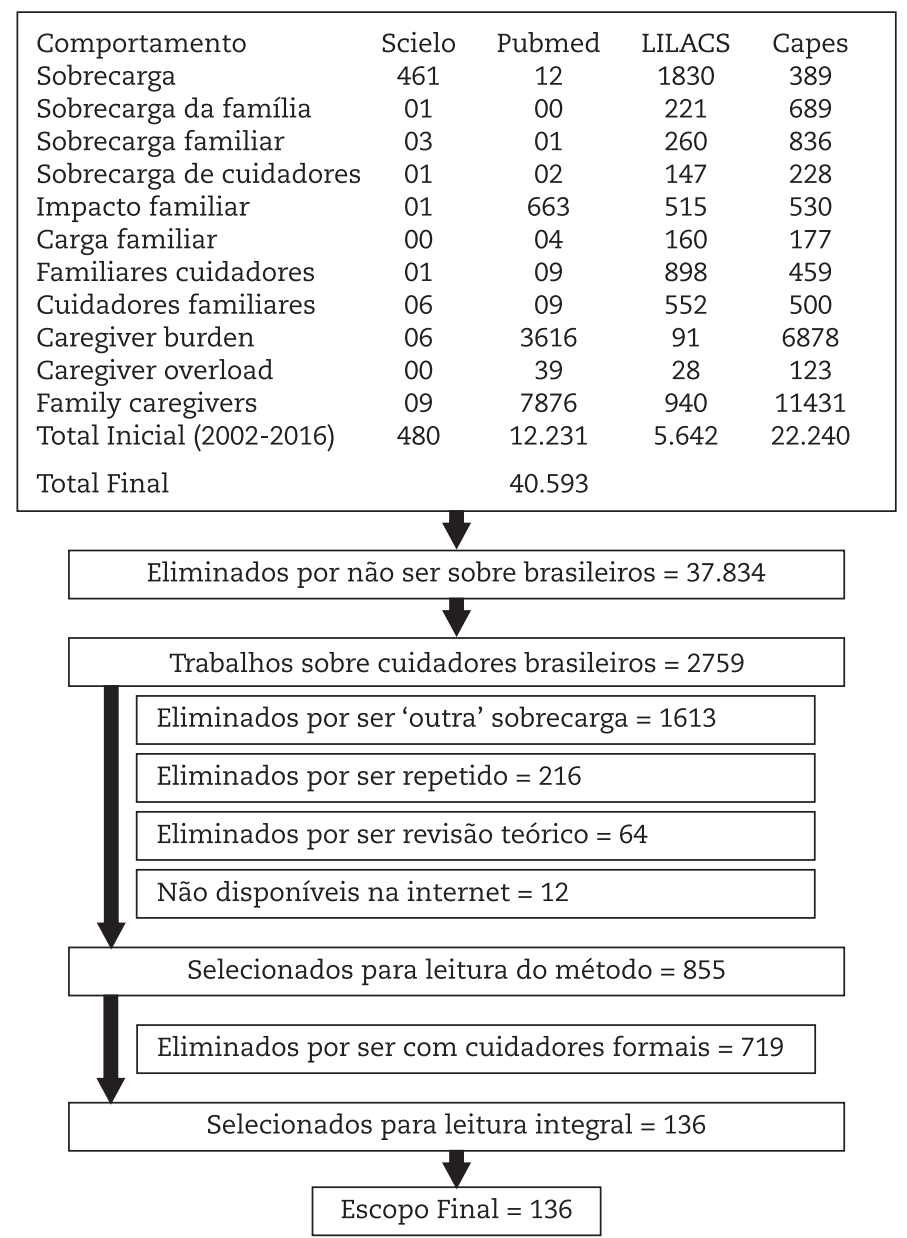

Figura 1. Percurso metodológico para seleção dos artigos 
Todos os estudos revisados eram transversais. A maioria eram pesquisas quantitativas $(n=101 ; 74,26 \%)$, enquanto os trabalhos qualitativos representaram $21,32 \%$ $(n=29)$ e apenas seis trabalhos $(4,41 \%)$ relatavam investigações mistas. Além disso, pôde-se observar um aumento progressivo no número de publicações dentro do período considerado. A média de artigos publicados por ano foi de 9,71 , tendo sido publicados apenas dois trabalhos no ano de 2002 (1,47\%), havendo um pico de estudos divulgados em 2012, ano de maior publicação de estudos, com 21 artigos (15,44\%) e, em 2016, foram publicados 17 trabalhos com essa temática (12,44\%) (Figura 2).

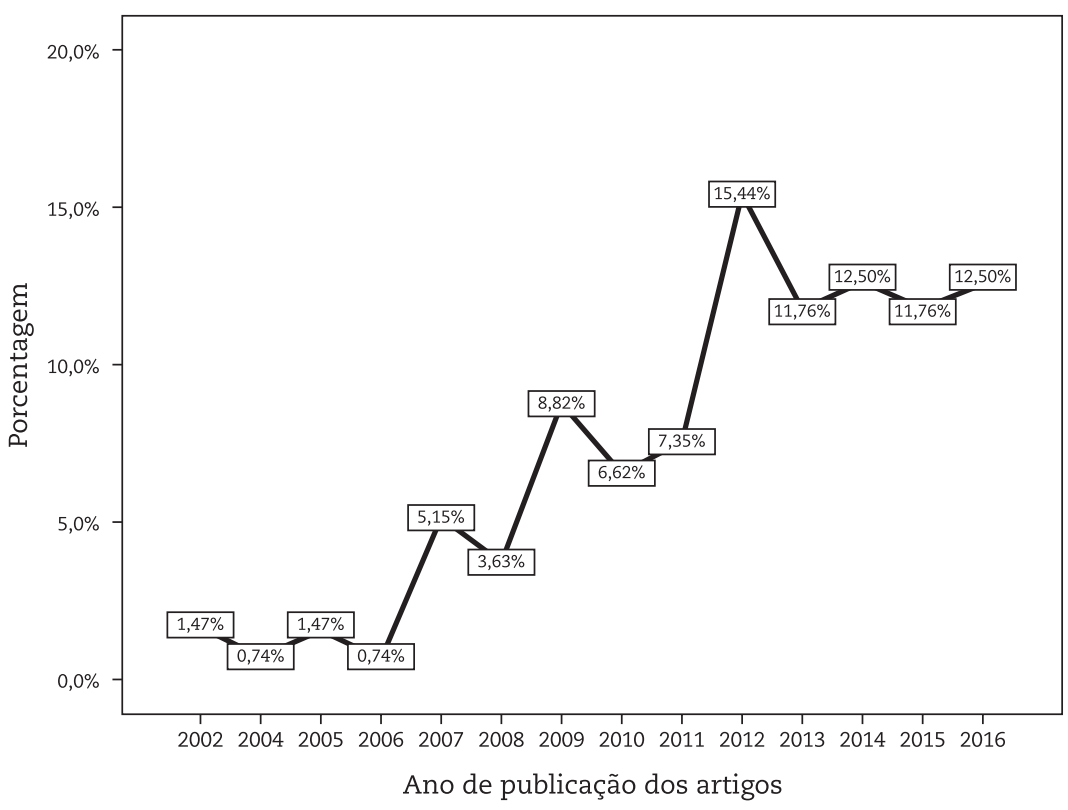

Figura 2. Distribuição das publicações sobre sobrecarga dos cuidadores por ano (2002 - 2016).

Foram localizados trabalhos publicados em 58 revistas no período considerado. As revistas que mais publicaram sobre o tema foram a "Ciência \& Saúde Coletiva" (n $=11$ artigos), a Revista de Enfermagem da USP $(n=10)$, a Revista Latino-Americana de Enfermagem $(n=9)$ e a Acta Paulista de Enfermagem $(n=8)$. A Universidade de São Paulo se destacou na produção de pesquisas sobre cuidadores informais, respondendo por 24 artigos $(17,64 \%)$ no período avaliado. Outras duas instituições, a Universidade Federal do Rio de Janeiro ( $n=11$ artigos) e a Universidade Federal do São João Del Rei $(n=08$ artigos) também se destacaram.

A maior parte dos estudos investigou amostras da região Sudeste $(n=75)$, nordeste $(n=26)$ ou sul $(n=25)$. Entre os trabalhos qualitativos, as amostras variaram entre 3 e 121 cuidadores. Nos estudos quantitativos, as amostras variaram entre 10 e 1164 participantes. Os trabalhos de metodologia mista contaram com amostras entre 17 e 208 cuidadores (Tabela 1).

Os motivos que geraram a necessidade de cuidado variaram, entre cuidadores eram responsáveis por pessoas com necessidades de cuidados físicos $(n=53)$, pessoas com transtornos psiquiátricos $(n=42)$ ou transtornos neuropsiquiátricos $(n=23)$. Entre as causas físicas de dependência foram epilepsia, paralisia cerebral, afasia, deficiência intelectual, idosos dependentes, acidente vascular encefálico, câncer, síndrome de Down, Transtorno do Espectro Autista, distrofia muscular, síndrome metabólica, AIDS, doenças crônicas, deficiência visual, lesão medular, trauma raquimedular e doença orofacial. Os principais transtornos psiquiátricos que geraram necessidade de um cuidador foram esquizofrenia, transtorno obsessivo-compulsivo e depressão. A demência, o Mal de Alzheimer, foram os principais problemas neuropsiquiátricos observados. Houve também 18 trabalhos $(13,20 \%)$ que avaliaram cuidadores de idosos sem doenças associadas, descritos como "idosos frágeis" ou "idosos dependentes".

Independentemente da causa, o perfil dos cuidadores foi similar nos estudos revisados. A maioria dos cuidadores era do sexo feminino, familiares (em especial mães) dos dependentes, acima de 60 anos e com menor escolarização. Os diversos estudos indicaram que os cuidadores precisavam lidar com tarefas e situações que, por vezes, extrapolavam seus recursos físicos, emocionais e financeiros. Repetidamente foram observados relatos sobre a necessidade que os cuidadores diminuíssem suas horas no trabalho ou parassem de trabalhar para cuidar do familiar (ex.: Quadros, Gigante, Kantorski, \& Jardim, 2012), sobre a falta de preparação 
dos familiares para entender as doenças e lidar com os comportamentos da pessoa sob seus cuidados (ex.: Camargos et al., 2012), sobre a perda de suporte social (ex.: Nars \& Kausar, 2009), dificuldades financeiras (ex.: Barroso et al., 2007) e, no caso dos cuidadores de pacientes psiquiátricos, sobre o sofrimento com o preconceito relacionado à doença mental (ex.: Ribeiro, Martins, \& Oliveira, 2009). A sobrecarga variou entre moderada e elevada, independentemente da forma de avaliação e da patologia das pessoas que recebiam cuidados. Os resultados mostraram, ainda, que quanto maior a dependência do paciente, maior o grau de sobrecarga dos cuidadores (Bianchi, Flesch, Alves, Batistoni, \& Neri, 2016).

Com relação às formas de avaliação, foram identificadas 16 técnicas/instrumentos para avaliação da sobrecarga. Um único instrumento foi utilizado em 130 pesquisas $(95,59 \%)$ e métodos combinados (entrevista + escala/diário de campo, observação + grupo focal ou duas escalas) foram utilizados em seis trabalhos. Entre as técnicas não validadas, a entrevista foi a mais utilizada ( $n=27)$, seguida dos questionários $(n=06)$, grupos focais $(n=04)$, história oral $(n=02)$, observação $(n=02)$, álbum de família $(n=01)$, diário de campo $(n=01)$ e história de vida $(n=01)$.

A escala mais utilizada foi a ZBI $(n=59$ trabalhos). Os outros instrumentos utilizados foram: FBIS $(n=19)$, Caregiver Burden Scale $(n=11)$, Questionário de Avaliação da Sobrecarga do Cuidador Informal $(n=04)$, Escala de Impacto Familiar $(n=02)$, Inventário Maslach de Burnout $(n=01)$, Caregiver Burden Inventory $(n=01)$, Caregiver Reaction Assessment $(n=01)$ e Inventário Neuropsiquiátrico de Desgaste $(n=01)$.

Tabela 1

Caracterização dos estudos revisados e exemplos por tipo de instrumento

\begin{tabular}{|c|c|c|c|c|}
\hline Instrumento & Período & $\begin{array}{c}\text { Total } \\
\text { artigos }\end{array}$ & Amostra & Exemplos de trabalhos \\
\hline Zarit Burden Interview & $2002-2016$ & 59 & $10-874$ & $\begin{array}{l}\text { Pedreira e Oliveira (2012), Misquiatti } \\
\text { et al. (2015), Moura et al. (2015) }\end{array}$ \\
\hline Entrevista & $2003-2016$ & 27 & $03-555$ & $\begin{array}{l}\text { Pedreira e Oliveira (2012), Almico } \\
\text { e Faro (2014), Machado et al. (2015) }\end{array}$ \\
\hline FBIS-BR & $2005-2013$ & 19 & $10-243$ & Borghi et al. (2013) \\
\hline Caregiver Burden Scale & $2011-2016$ & 11 & $17-160$ & Marcon et al. (2012) \\
\hline Questionário & $2009-2016$ & 06 & $41-1164$ & Treichel et al. (2016) \\
\hline $\begin{array}{l}\text { Questionário de Avaliação de } \\
\text { Sobrecarga do Cuidador Informal }\end{array}$ & $2013-2015$ & 03 & $12-132$ & Souza et al. (2015) \\
\hline Grupo Focal & 2014 & 02 & $08-115$ & Delgado (2014) \\
\hline História Oral & $2009-2014$ & 02 & $04-14$ & \\
\hline Observação & $2012-2014$ & 02 & $06-115$ & Delgado (2014) \\
\hline Álbum de família & 2009 & 01 & 03 & Silva e Santos (2009) \\
\hline Escala de Impacto Familiar & 2009 & 01 & 123 & Goursand et al. (2009) \\
\hline Inventário Maslach de Burnout & 2011 & 01 & 145 & Valente et al. (2011) \\
\hline Inventário de Sobrecarga do Cuidador & 2015 & 01 & 120 & Valer et al. (2015) \\
\hline Caregiver Reaction Assessment & 2015 & 01 & 30 & Mota et al. (2015) \\
\hline Diário de Campo & 2012 & 01 & 08 & Pedreira e Oliveira (2012) \\
\hline História de Vida & 2016 & 01 & 06 & Souza et al. (2015) \\
\hline $\begin{array}{l}\text { Inventário Neuropsiquiátrico } \\
\text { de Desgaste }\end{array}$ & 2016 & 01 & 96 & Storti et al. (2016) \\
\hline
\end{tabular}




\section{Discussão}

Os resultados permitiram identificar 16 técnicas de avaliação da sobrecarga de cuidadores informais no Brasil, utilizadas no período entre 2002 e 2016. Dessas, nove eram escalas internacionais, validadas para o contexto brasileiro. Cassepp-Borges, Balbinotti e Teodoro (2010) indicaram que há um maior número de instrumentos criados em contexto internacional e adaptados para o Brasil pela maior rapidez com que esse tipo de processo ocorre, quando comparado à criação de um novo instrumento. Sete técnicas qualitativas ou quantitativas não validadas também foram identificadas. As entrevistas e questionários foram criados especificamente para os estudos em que foram utilizadas, variando quanto aos aspectos abordados e tipos de perguntas. Todas incluíam perguntas sobre o perfil dos cuidadores e sua percepção sobre as dificuldades envolvidas em cuidar de alguém com uma necessidade de cuidados especiais.

Nenhum dos instrumentos identificados possui ponto de corte definido para o Brasil e a maioria deles avalia a sobrecarga subjetiva dos cuidadores. A escala mais utilizada, a ZBI, foi criada para avaliar os sentimentos negativos dos cuidadores de familiares de pacientes com demência (Zarit, Orr, \& Zarit, 1985). Apesar de ter sido elaborada para cuidadores de pacientes com demência, a ZBI tem sido utilizada para avaliar cuidadores de pessoas com diversos diagnósticos. Internacionalmente, o ponto de corte dessa escala foi estabelecido em 24 pontos para identificação da sobrecarga elevada, potencialmente relacionada ao surgimento de transtornos depressivos (Schreiner et al., 2006), mas a versão brasileira da ZBI não possui ponto de corte estabelecido. Dos instrumentos de avaliação da sobrecarga, apenas a FBIS avalia a sobrecarga objetiva - frequência de atividades realizadas e a sobrecarga subjetiva dos cuidadores (Bandeira, Calzavara, Freitas, \& Barroso, 2007; Bandeira, Calzavara, \& Castro, 2008). Esse instrumento também não possui ponto de corte estabelecido para o Brasil.

Foram encontrados trabalhos que utilizaram a Caregiver Burden Scale, validada para o Brasil em 2015(Valer, Aires, Fengler, \& Paskulin, 2015), mas já era utilizada no Brasil antes disso (Marcon, Rubira, Espinosa, Belasco, \& Barbosa, 2012). O Inventário Neuropsiquiátrico de
Desgaste foi um módulo complementar anexado ao Inventário Neuropsiquiátrico para avaliar o desgaste associado aos sintomas psiquiátricos dos idosos e se encontra validado para o Brasil (Camozzato, Kochhann, Simeoni, \& Chaves, 2008; Camozzato, Godinho, Kochhann, Massochini, \& Chaves, 2015). O instrumento também não tem ponto de corte estabelecido. A Escala de Impacto Familiar foi criada para avaliação da sobrecarga de pais de crianças com problemas orofaciais e foi validada para o Brasil por Barbosa e Gavião (2009). Mas após sua validação, apenas um estudo foi localizado fazendo uso desse instrumento (Goursand et al., 2009). Cabe destacar que o Inventário Maslach de Burnout, utilizado em um estudo como forma de avaliar a sobrecarga, não avalia esse construto, e sim burnout (Valente et al., 2011).

O perfil dos cuidadores também foi considerado e mostrou que essa função é prioritariamente feminina, sobrecarregados, que vivenciavam dificuldades financeiras, no manejo dos pacientes, de suas crises e sentiam o impacto de ser cuidador em sua qualidade de vida. Esse perfil é muito similar ao descrito em estudos internacionais, levantando questões sobre um possível perfil universal dos cuidadores informais (Nars \& Kausar, 2009). A percepção que cuidadores de pessoas com diversas patologias sofrem com sobrecarga mostram a necessidade de conduzir a mudanças nas políticas públicas para essa população, que incluam serviços de apoio aos familiares, equipes domiciliares para auxiliar com orientações e no manejo de crises, cursos de capacitação básica, além de criar programas de acompanhamento médico, psicoterápico e fisioterápico para os familiares que apresentam problemas físicos ou emocionais.

Considerando a importância dos cuidadores informais para o cuidado das pessoas com problemas físicos ou psiquiátricos, fica evidenciada a importância de investir em pesquisas populacionais e longitudinais para compreender as reais condições de vida e as necessidades desses cuidadores, adotando instrumentos validados em sua condução, para aumentar a confiabilidade de seus resultados. Investigações sobre as reais carências dos familiares, utilizando instrumentos validados na avaliação, poderão embasar intervenções específicas mais bem-sucedidas, contribuindo para a diminuição da sobrecarga e a melhoria da qualidade de vida dos cuidadores informais brasileiros.

\section{Referências}

Almeida, M. M., Schal, V. T., Martins, A. M., \& Modena, C. M. (2010). A sobrecarga de cuidadores de pacientes com esquizofrenia. Revista de Psiquiatria do Rio Grande de Sul, 32(3), 73-79. doi: 10.1590/S0101-81082010005000003

Almico, T., \& Faro, A. (2014). Enfrentamento de cuidadores de crianças com câncer em processo de quimioterapia. Revista Psicologia, Saúde E Doenças, 15(3), 723-737. doi: 10.15309/14psd150313

Bandeira, M., Calzavara, M. G. P., \& Castro, I. (2008). Estudo de validade da escala de sobrecarga de familiares cuidadores de pacientes psiquiátricos. Jornal Brasileiro de Psiquiatria, 57(2), 98-104. doi: 10.1590/S0047-20852008000200003 
Bandeira, M., Calzavara, M. G. P., Freitas, L. C., \& Barroso, S. M. (2007). Escala de sobrecarga de familiares de pacientes psiquiátricos (FBISBR): Estudo de confiabilidade da versão brasileira. Revista Brasileira de Psiquiatria, 29(1), 1-5. doi: 10.1590/S1516-44462006005000015

Barbosa, T. S., \& Gavião, M. B. D. (2009). Evaluation of the family impact scale for use in Brazil. Journal of Applied Oral Science, 17(5), 397403. doi: 10.1590/S1678-77572009000500009

Barroso, S. M., Bandeira, M., \& Nascimento, E. (2007). Sobrecarga de familiares de pacientes psiquiátricos atendidos na rede pública. Archives of Clinical Psychiatry (São Paulo), 34(6), 270-277. doi: 10.1590/S0101-60832007000600003

Bianchi, M, Flesch, L. D., Alves, E. V. C., Batistoni, S. S. T., \& Neri, A. L. (2016). Zarit burden interview psychometric indicators applied in older people caregivers of other elderly. Revista Latino-Americana de Enfermagem, 24, 1-9. doi: 10.1590/1518-8345.1379.2835

Borghi, A. C., Castro, V. C., Marcon, S. S., \& Carreira, L. (2013). Sobrecarga de familiares cuidadores de idosos com doença de Alzheimer: Um estudo comparativo. Revista Latino-Americana de Enfermagem, 21(4), 876-883. doi: 10.5935/1414-8145.20160047

Camargos, E. F., Souza, A. B., Nascimento, A. S., Morais-e-Silva, A.C., Quintas, J. L.,... Louzada, L. L. (2012). Use of psychotropic medications by caregivers of elderly patients with dementia: is this a sign of caregiver burden? Arquivos de Neuro-Psiquiatria, 70(3), 169174. doi: 10.1590/S0004-282X2012000300003

Camozzato, A. L., Godinho, C., Kochhann, R., Massochini, G., \& Chaves, M. L. (2015). Validity of the Brazilian version of the neuropsychiatric inventory questionnaire (NPI-Q). Arquivos de Neuro-Psiquiatria, 73(1), 41-45. doi: 10.1590/0004-282X20140177

Camozzato, A. L.., Kochhann, R., Simeoni, C, Konrath, C. A., Franz, A. P., Carvalho, A., \& Chaves, M. L. (2008). Reliability of the Brazilian Portuguese version of the Neuropsychiatric Inventory (NPI) for patients with Alzheimer's disease and their caregivers. International Psychogeriatrics, 20(02), 383-393. doi: 10.1017/S1041610207006254

Cassepp-Borges, V., Balbinotti, M . A., \& Teodoro, M. L. (2010). Tradução e validação de conteúdo: Uma proposta para a adaptação de instrumentos. Em: L. Pasquali (Ed.). Instrumentação psicológica: Fundamentos e práticas (pp. 506-520). Porto Alegre: Artmed.

Delgado, P. G. (2014). Sobrecarga do cuidado, solidariedade e estratégia de lida na experiência de familiares de Centros de Atenção Psicossocial. Physis: Revista de Saúde Coletiva, 24(4). 1103-1126. doi: 10.1590/S0103-73312014000400007.

Galera, S. A. F., Zanetti, A. C.G, Ferreira, G. C. S., Giacon, B. C. C., \& Cardoso, L. (2011). Pesquisas com famílias de portadores de transtorno mental. Revista Brasileira de Enfermagem, 64(4), 774-778. doi: 10.1590/S0034-71672011000400022

Goursand, D., Paiva, S. M., Zarzar, P. M., Pordeus, I. A., \& Allison, P. J. (2009). Family Impact Scale (FIS): Psychometric properties of the Brazilian Portuguese language version. European Journal of Paediatric Dentistry, 10(3), 141-146. Recuperado de: http://admin.ejpd.eu/ download/2009-03-07.pdf

Hedler, H. C., Santos, M. J. S., Faleiros, V. P., \& Almeida, M. A. A. (2016). Representação social do cuidado e do cuidador familiar do idoso. Revista Katál, 19(1), 143-153. doi: 10.1590/1414-49802016.00100015

Machado, W. C., Alvarez, A. B., Teixeira, M. L. O., Branco, M. S. C., \& Figueiredo, N. M. A. (2015). Como cuidadores de paraplégicos lidam com sobrecarga de atividades no dia a dia. Revista Online de Pesquisa Cuidado é Fundamental, 7(1), 1796-1807. doi: 10.9789/21755361.2015.v7i1.1796-1807

Marcon, S. R., Rubira, E. A., Espinosa, M. M., Belasco, A., \& Barbosa, D. A. (2012). Qualidade de vida e sobrecarga de cuidados em cuidadores de dependentes químicos. Acta Paulista de Enfermagem, 25(2), 7-12. Recuperado de http://www.redalyc.org/articulo. oa?id=307026829007

Medeiros, M. M. D. C., Ferraz, M. B., Quaresma, M. R., \& Menezes, A. P. D. (1998). Adaptação ao contexto cultural brasileiro e validação do "Caregiver Burden Scale". Revista Brasileira de Reumatologia, 38(4), 193-199.

Misquiatti A. R. N., Brito, M. C., Ferreira, F. T. S., \& Assumpção Junior, F. B. (2015). Sobrecarga familiar e crianças com transtornos do espectro do autismo:_erspectiva dos cuidadores. Revista CEFAC, 17(1), 192-200. doi: 10.1590/1982-0216201520413

Mota, F. R. N., Victor, J. F., Silva, M. J., Bessa, M. E. P., Amorim, V. L.,... Cavalcante, M. L. S. N. (2015). Adaptação transcultural do Caregiver Reaction Assessment para uso no Brasil com cuidadores informais de idosos. Revista da Escola de Enfermagem da USP, 49(3), 424-431. doi: 10.1590/S0080-623420150000300010

Moura, M. C. D. S., Wutzki, H. C., Voos, M. C., Resende, M. B. D., Reed, U. C., \& Hasue, R. H. (2015). Is functional dependence of Duchenne muscular dystrophy patients determinant of the quality of life and burden of their caregivers? Arquivos de Neuro-Psiquiatria, 73(1), 52-57. doi: 10.1590/0004-282X20140194

Nars, T., \& Kausar, R. (2009). Psychoeducation and the family burden in schizophrenia: a randomized controlled Trial. Annals of General Psychiatry, 8, 17-23. doi: 10.1186/1744-859X-8-17

Pedreira, L. C., \& Oliveira, M. A. S. (2012). Cuidadores de idosos dependentes no domicílio: mudanças nas relações familiares. Revista Brasileira de Enfermagem, 65(5), 730-736. doi: 10.1590/S0034-71672012000500003

Quadros, L. C. M., Gigante, D. P., Kantorski, L. P., \& Jardim, V. M. R. (2012). Transtornos psiquiátricos menores em cuidadores familiares de usuários de Centros de Atenção Psicossocial do Sul do Brasil. Cadernos de Saúde Pública, 28(1), 95-103. doi: 10.1590/S0102311X2012000100010

Ribeiro, M. B. S., Martins, S. T. F., \& Oliveira, L. R. (2009). Familiares de usuários vivenciando a transformação do modelo assistencial psiquiátrico. Estudos de Psicologia, 14(2), 133-140. doi: 10.1590/S1413-294X2009000200006

Schreiner, A. S., Morimoto, T., Arai, Y., Zarit, S. H. (2006). Assessing family caregiver's mental health using a statistically derived cut-off for the Zarit Burden Interview. Aging \& Mental heath, 10(2), 107-111. doi: 10.1080/13607860500312142

Silva G., \& Santos, M. A. (2009). Álbum de família e esquizofrenia: convivência em retrato. Psicologia em Estudo, 14(1), 83-91. doi: 10.1590/ S1413-73722009000100011

Souza, L. R., Hanus, J. S., Libera, D., Bolzan, L., Silva, V. M.,... Mangilli, E. M. (2015). Sobrecarga no cuidado, estresse e impacto na qualidade de vida de cuidadores domiciliares assistidos na atenção básica. Cadernos Saúde Coletiva, 23(2). doi: 10.1590/1414462X201500020063.

Storti, L. B., Quintino, D. T., Silva, N. M., Kusumota, L., \& Marques, S. (2016). Neuropsychiatric symptoms of the elderly with Alzheimer's disease and the family caregivers' distress. Revista Latino-Americana de Enfermagem, 24, e2751. Epub 15 de agosto de 2016. doi: .https:// dx.doi.org/10.1590/1518-8345.0580.2751

Treichel, C. A. S., Jardim, V. M. R., Kantorski, L. P., Vasem, M. L., \& Neutzling, A. S. (2016). Clustering of minor psychiatric disorders and burden among family caregivers of individuals with mental illness. Ciência E Saúde Coletiva, 21(2), 585-590. doi: 10.1590/141381232015212.04392015 
Valente, L. E. Truzzi, A., Souza, W. F., Alves, G. S., Alves, C. E. O., Sudo, F. K. et al. (2011). Health self-perception by dementia family caregivers: Sociodemographic and clinical factors. Arquivos de Neuro-Psiquiatria, 69(5), 739-744. doi: 10.1590/S0004-282X2011000600003

Valer, D. B, Aires, M., Fengle, F. L., \& Paskulin, L. M. G. (2015). Adaptação e validação do Inventário de Sobrecarga do Cuidador para uso em cuidadores de idosos. Revista Latino-Americana de Enfermagem, 23(1), 130-1388. doi: 10.1590/0104-1169.3357.2534

Whalen, K. J., \& Buchholz, S. W. (2009). The Reliability, Validity and Feasibility of Tools Used to Screen for Caregiver Burden: a Systematic Review. JBI Library of Sistematic Review, 7(32),1373-1430. doi: 10.11124/01938924-200907320-00001

Wijngaarden, B., \& Schene, A. H. (2010). Measuring family or carer burden in severe mental illness: The instruments. Em M. Tansella, \& G. Thornicroft (Eds.), Mental Health Outcome Measures (3a ed.). (pp.116-135). Londres: Gaskell.

Zarit, S. H., Orr, N. K., \& Zarit, J. M. (1985). The hidden victims of Alzheimer's disease; families under stress. New York: New York University.

\section{Sobre os autores}

Sabrina M. Barroso é psicóloga, doutora em Saúde Pública e professora da Universidade Federal do Triângulo Mineiro.

Luísa Parreira Santos é psicóloga, mestranda no Programa de Pós-graduação em Psicologia da Universidade Federal do Triângulo Mineiro e atua no CAPS em Uberlândia.

Luciana Francielle e Silva é psicóloga, mestranda no Programa de Pós-graduação em Psicologia da Universidade Federal do Triângulo Mineiro. 